\title{
Direct product of finite anti fuzzy normal sub-rings over non-associative rings
}

\author{
Nasreen Kausar ${ }^{\mathrm{a}, *}$, Mohammad Munir ${ }^{\mathrm{b}}$, Salahuddin ${ }^{\mathrm{c}}$, Zuhairi Baharudin ${ }^{\mathrm{d}}$, Badar UI Islam ${ }^{\mathrm{e}}$ \\ ${ }^{a}$ Department of Mathematics and Statistics, University of Agriculture, Faisalabad, Pakistan. \\ ${ }^{b}$ Department of Mathematics, Government Postgraduate College, Abbottabad, Pakistan. \\ ${ }^{c}$ Department of Mathematics, Jazan University, Jazan, Kingdom of Saudi Arabia. \\ ${ }^{d}$ Department of Electrical and Electronics Engineering, University of Teknologi Petronas, Seri Iskandar, Malaysia. \\ ${ }^{e}$ Department of Electrical Engineering, NFC IEFR FSD, Pakistan.
}

\begin{abstract}
In this paper, we define the concept of direct product of finite anti fuzzy normal sub-rings over non-associative and noncommutative rings LA-rings and investigate the some fundamental properties of direct product of anti fuzzy normal subrings.
\end{abstract}

Keywords: Direct product of fuzzy sets, anti fuzzy LA-sub-rings, anti fuzzy normal LA-sub-rings

2020 MSC: 03F55, 08A72, 20N25

(C)2021 All rights reserved.

\section{Introduction}

In 1972, a generalization of commutative semigroups was established by Kazim et al. [21]. In ternary commutative law: $a b c=c b a$, they introduced the braces on the left side of this law and explored a new pseudo associative law, that is: $(a b) c=(c b) a$. This law $(a b) c=(c b) a$ called the left invertive law. A groupoid $S$ is said to be a left almost semigroup (abbreviated as LA-semigroup) if it satisfies the left invertive law: $(a b) c=(c b) a$.

In [12] (resp. [7]), a groupoid $S$ is said to be medial (resp. paramedial) if $(a b)(c d)=(a c)(b d)$ (resp. $(a b)(c d)=(d b)(c a))$. In [21], an LA-semigroup is medial, but in general an LA-semigroup needs not to be paramedial. Every LA-semigroup with left identity is paramedial by Protic et al. [28] and also satisfies $a(b c)=b(a c),(a b)(c d)=(d c)(b a)$.

Kamran [14], extended the notion of LA-semigroup to the left almost group (LA-group). An LAsemigroup $\mathrm{G}$ is said to be a left almost group, if there exists left identity $e \in G$ such that $e a=a$ for all $a \in G$, and for every $a \in G$ there exists $b \in G$ such that $b a=e$.

\footnotetext{
*Corresponding author

Email addresses: kausar.nasreen57@gmail.com (Nasreen Kausar), dr.mohammadmunir@gmail .com (Mohammad Munir)

doi: $10.22436 /$ jmcs.022.04.08
}

Received: 2020-07-12 Revised: 2020-08-08 Accepted: 2020-08-17 
Shah et al. [31], discussed the left almost ring (LA-ring) of finitely nonzero functions which is a generalization of commutative semigroup ring. By a left almost ring, we mean a non-empty set $R$ with at least two elements such that $(R,+)$ is an LA-group, $(R, \cdot)$ is an LA-semigroup, both left and right distributive laws hold. For example, from a commutative ring $(R,+, \cdot)$, we can always obtain an LA-ring $(R, \oplus, \cdot)$ by defining for all $a, b \in R, a \oplus b=b-a$ and $a \cdot b$ is same as in the ring. In fact an LA-ring is non-associative and non-commutative ring.

A non-empty subset $A$ of an LA-ring $R$ is called an LA-sub-ring of $R$ if $a-b$ and $a b \in A$ for all $a, b \in A$. $A$ is called a left (resp. right) ideal of $R$ if $(A,+)$ is an LA-group and $R A \subseteq A$ (resp. $A R \subseteq A$ ). $A$ is called an ideal of $R$ if it is both a left ideal and a right ideal of $R$.

First time, the concept of fuzzy set introduced by Zadeh in his classical paper [34]. This concept has provided a useful mathematical tool for describing the behavior of systems that are too complex to admit precise mathematical analysis by classical methods and tools. Extensive applications of fuzzy set theory have been found in various fields such as artificial intelligence, computer science, management science, expert systems, finite state machines, Languages, robotics, coding theory and others.

Liu [24], introduced the concept of fuzzy sub-rings and fuzzy ideals of a ring. Many authors have explored the theory of fuzzy rings (for example [8-10, 22, 25, 26, 33]). Gupta et al. [10], gave the idea of intrinsic product of fuzzy subsets of a ring. Kuroki [22], characterized regular (intra-regular, both regular and intra-regular) rings in terms of fuzzy left (right, quasi, bi-) ideals.

Biswas [6], introduced the concept of anti fuzzy subgroups and studied the basic properties of groups in terms of such ideals. Hong and Jun [11], modified the Biswas idea and applied it into BCK-algebra. Akram and Dar defined anti fuzzy left h-ideals of a hemiring and discussed the basic properties of a hemiring in [4].

Sherwood [32], introduced the concept of product of fuzzy subgroups. After this, further study on this concept continued by Osman [1, 2] and Ray [29]. Zaid [3], gave the idea of normal fuzzy subgroups.

Shal et al. [30], originated the studied of intuitionistic fuzzy normal LA-sub-rings over left almostring. Islam et al. [17] initiated the intuitionistics fuzzy ideals with thresholds $(\alpha, \beta]$ in left almost ring. Javaid et al. [18], also studied the left almost rings by fuzzy ideals. Waqar et al. [19], studied the left almost rings by using the intuitionistic fuzzy bi-ideals. Kausar et al. [15], explored the direct product of finite intuitionistic anti fuzzy normal LA-sub-rings over LA-rings. Waqar et al. [20], investigated the direct product of finite fuzzy normal LA-sub-rings on Left Almost-rings.

Recently Munir et al. [27], discussed on the prime fuzzy m-bi ideals in semigroups.

In this paper, we define the concept of direct product of anti fuzzy normal LA-sub-rings. In Section 2, we investigate the some basic properties of anti fuzzy normal LA-sub-rings of an LA-ring R. Section 3 , we define the direct product of fuzzy subsets $\mu_{1}, \mu_{2}$ of LA-rings $R_{1}, R_{2}$, respectively and investigate the some elementary properties of direct product of anti fuzzy normal LA-sub-rings of an LA-ring $R_{1} \times$ $R_{2}$. In Section 4 , we define the direct product of fuzzy subsets $\mu_{1}, \mu_{2}, \ldots, \mu_{n}$ of LA-rings $R_{1}, R_{2}, \ldots, R_{n}$, respectively and examine the some fundamental properties of direct product of anti fuzzy normal LAsub-rings of an LA-ring $R_{1} \times R_{2} \times \cdots \times R_{n}$. Specifically we show the following.

1. Let $A$ and $B$ are two LA-sub-rings of an LA-ring $R$. Then $A \cap B$ is an LA-sub-ring of $R$ if and only if the anti characteristic function $\chi_{Z}^{C}$ of $Z=A \cap B$ is an anti fuzzy normal LA-sub-ring of $R$.

2. Let $X=A \times B$ and $Y=C \times D$ are two LA-sub-rings of an LA-ring $R_{1} \times R_{2}$. Then $X \cap Y$ is an LA-subring of $R_{1} \times R_{2}$ if and only if the anti characteristic function $\chi_{Z}^{C}$ of $Z=X \cap Y$ is an anti fuzzy normal LA-sub-ring of $R_{1} \times R_{2}$.

3. Let $A=A_{1} \times A_{2} \times \cdots \times A_{n}$ and $B=B_{1} \times B_{2} \times \cdots \times B_{n}$ are two LA-sub-rings of an LA-ring $R_{1} \times$ $R_{2} \times \cdots \times R_{n}$. Then $A \cap B$ is an LA-sub-ring of $R_{1} \times R_{2} \times \cdots \times R_{n}$ if and only if the anti characteristic function $\chi_{Z}^{C}$ of $Z=A \cap B$ is an anti fuzzy normal LA-sub-ring of $R_{1} \times R_{2} \times \cdots \times R_{n}$.

\section{Anti fuzzy normal LA-sub-rings}

In this section, we investigate the some basic properties of anti fuzzy normal LA-sub-rings of an 


\section{LA-ring R.}

By a fuzzy subset $\mu$ of an LA-ring $R$, we mean a function $\mu: R \rightarrow[0,1]$ and the complement of $\mu$ is denoted by $\mu^{\prime}$, is a fuzzy subset of $R$ defined by $\mu^{\prime}(x)=1-\mu(x)$ for all $x \in R$.

A fuzzy subset $\mu$ of an LA-ring $R$ is an anti fuzzy LA-sub-ring of $R$ if $\mu(x-y) \leqslant \max \{\mu(x), \mu(y)\}$ and $\mu(x y) \leqslant \max \{\mu(x), \mu(y)\}$ for all $x, y \in R$.

An anti fuzzy LA-sub-ring of an LA-ring $R$ is said to be an anti fuzzy normal LA-sub-ring of $R$ if $\mu(x y)=\mu(y x)$ for all $x, y \in R$.

Let $A$ is a non-empty subset of an LA-ring $R$. The anti characteristic function of $A$ is denoted by $\chi_{\AA}^{C}$ and defined by

$$
\chi_{\AA}^{C}: R \rightarrow[0,1] \mid x \rightarrow \chi_{\AA}^{C}(x)= \begin{cases}0, & \text { if } x \in A, \\ 1, & \text { if } x \notin A .\end{cases}
$$

Lemma 2.1. Let $\mathrm{A}$ is a non-empty subset of an LA-ring $\mathrm{R}$. Then $\mathrm{A}$ is an LA-sub-ring of $\mathrm{R}$ if and only if the anti characteristic function $\chi_{\AA}^{C}$ of $A$ is an anti fuzzy normal $L A$-sub-ring of $R$.

Proof. Let $A$ is an LA-sub-ring of $R$ and $a, b \in R$. If $a, b \in A$, then by definition of anti characteristic function $\chi_{\AA}^{C}(a)=0=\chi_{\AA}^{C}(b)$. Since $a-b, a b \in A, A$ is an LA-sub-ring of $R$. This implies that

$$
\chi_{\AA}^{C}(a-b)=0=0 \vee 0=\chi_{\AA}^{C}(a) \vee \chi_{\AA}^{C}(b) \quad \text { and } \quad \chi_{\AA}^{C}(a b)=0=0 \vee 0=\chi_{\AA}^{C}(a) \vee \chi_{\AA}^{C}(b) .
$$

Thus $\chi_{\AA}^{C}(a-b) \leqslant \max \left\{\chi_{\AA}^{C}(a), \chi_{\AA}^{C}(b)\right\}$ and $\chi_{\AA}^{C}(a b) \leqslant \max \left\{\chi_{\AA}^{C}(a), \chi_{\AA}^{C}(b)\right\}$. Since $a b$ and $b a \in A$, so $\chi_{\AA}^{C}(a b)=0=\chi_{\AA}^{C}(b a)$, i.e., $\chi_{\AA}^{C}(a b)=\chi_{\AA}^{C}(b a)$. Similarly we have

$$
\chi_{\AA}^{C}(a-b) \leqslant \max \left\{\chi_{\AA}^{C}(a), \chi_{\AA}^{C}(b)\right\}, \quad \chi_{\AA}^{C}(a b) \leqslant \max \left\{\chi_{\AA}^{C}(a), \chi_{\AA}^{C}(b)\right\}, \quad \chi_{\AA}^{C}(a b)=\chi_{\AA}^{C}(b a),
$$

when $a, b \notin A$. Hence the anti characteristic function $\chi_{\AA}^{C}$ of $A$ is an anti fuzzy normal LA-sub-ring of $R$.

Conversely, suppose that the anti characteristic function $\chi_{\AA}^{C}$ of $A$ is an anti fuzzy normal LA-sub-ring of $R$. Let $a, b \in A$, then by definition $\chi_{\AA}^{C}(a)=0=\chi_{\AA}^{C}(b)$. By our supposition

$$
\chi_{\AA}^{C}(a-b) \leqslant \chi_{A}^{C}(a) \vee \chi_{A}^{C}(b)=0 \vee 0=0 \quad \text { and } \quad \chi_{A}^{C}(a b) \leqslant \chi_{A}^{C}(a) \vee \chi_{A}^{C}(b)=0 \vee 0=0 .
$$

Thus $\chi_{\AA}^{C}(a-b)=0=\chi_{\AA}^{C}(a b)$, i.e., $a-b, a b \in A$. Hence $A$ is an LA-sub-ring of $R$.

Lemma 2.2. If $\mathrm{A}$ and $\mathrm{B}$ are two $L A$-sub-rings of an $L A$-ring $\mathrm{R}$, then their intersection $\mathrm{A} \cap \mathrm{B}$ is also an $L A$-sub-ring of $R$.

Proof. Straight forward.

Proposition 2.3. Let $\mathrm{A}$ and $\mathrm{B}$ are two LA-sub-rings of an LA-ring $\mathrm{R}$. Then $\mathrm{A} \cap \mathrm{B}$ is an LA-sub-ring of $\mathrm{R}$ if and only if the anti characteristic function $\chi_{Z}^{C}$ of $Z=A \cap B$ is an anti fuzzy normal $L A$-sub-ring of $R$.

Proof. Let $Z=A \cap B$ is an LA-sub-ring of $R$ and $a, b \in R$. If $a, b \in Z=A \cap B$, then by definition of anti characteristic function $\chi_{Z}^{C}(a)=0=\chi_{Z}^{C}(b)$. Since $a-b, a b \in A, B, A$ and $B$ are LA-sub-rings of $R$. This implies that

$$
\chi_{Z}^{C}(a-b)=0=0 \vee 0=\chi_{Z}^{C}(a) \vee \chi_{Z}^{C}(b) \quad \text { and } \quad \chi_{Z}^{C}(a b)=0=0 \vee 0=\chi_{Z}^{C}(a) \vee \chi_{Z}^{C}(b) .
$$

Thus $\chi_{Z}^{C}(a-b) \leqslant \max \left\{\chi_{Z}^{C}(a), \chi_{Z}^{C}(b)\right\}$ and $\chi_{Z}^{c}(a b) \leqslant \max \left\{\chi_{Z}^{C}(a), \chi_{Z}^{C}(b)\right\}$. As ab and $b a \in Z$, so $\chi_{Z}^{C}(a b)=$ $0=\chi_{Z}^{C}(b a)$, i.e., $\chi_{Z}^{C}(a b)=\chi_{Z}^{C}(b a)$. Similarly we have

$$
\chi_{Z}^{C}(a-b) \leqslant \max \left\{\chi_{Z}^{C}(a), \chi_{Z}^{C}(b)\right\}, \quad \chi_{Z}^{C}(a b) \leqslant \max \left\{\chi_{Z}^{C}(a), \chi_{Z}^{C}(b)\right\}, \quad \chi_{Z}^{C}(a b)=\chi_{Z}^{C}(b a),
$$

when $a, b \notin Z$. Hence the anti characteristic function $\chi_{Z}^{C}$ of $Z$ is an anti fuzzy normal LA-sub-ring of $R$.

Conversely, assume that the anti characteristic function $\chi_{Z}^{C}$ of $Z=A \cap B$ is an anti fuzzy normal LA-sub-ring of $R$. Let $a, b \in Z=A \cap B$, this means that $\chi_{Z}^{C}(a)=0=\chi_{Z}^{C}(b)$. By our assumption

$$
\chi_{Z}^{C}(a-b) \leqslant \chi_{Z}^{C}(a) \vee \chi_{Z}^{C}(b)=0 \vee 0=0 \quad \text { and } \quad \chi_{Z}^{C}(a b) \leqslant \chi_{Z}^{C}(a) \vee \chi \cdot Z_{Z}^{C}(b)=0 \vee 0=0 .
$$

Thus $\chi_{Z}^{C}(a-b)=0=\chi_{Z}^{C}(a b)$, i.e., $a-b$ and $a b \in Z$. Hence $Z$ is an LA-sub-ring of $R$. 
Corollary 2.4. Let $\left\{A_{i}\right\}_{i \in I}$ is a family of LA-sub-rings of an LA-ring $\mathrm{R}$, then $\mathrm{A}=\cap \mathrm{A}_{i}$ is an LA-sub-ring of $\mathrm{R}$ if and only if the anti characteristic function $\chi_{A}^{C}$ of $A=\cap A_{i}$ is an anti fuzzy normal LA-sub-ring of $R$.

Lemma 2.5. If $\mu$ and $\gamma$ are two anti fuzzy normal $L A$-sub-rings of an $L A$-ring $R$, then their union $\mu \cup \gamma$ is also an anti fuzzy normal LA-sub-ring of $\mathrm{R}$.

Proof. Let $\mu$ and $\gamma$ are two anti fuzzy normal LA-sub-rings of an LA-ring R. We have to show that $\beta=\mu \cup \gamma$ is also an anti fuzzy normal LA-sub-ring of R. Now

$$
\begin{aligned}
\beta\left(z_{1}-z_{2}\right) & =(\mu \cup \gamma)\left(z_{1}-z_{2}\right) \\
& =\max \left\{\mu\left(z_{1}-z_{2}\right), \gamma\left(z_{1}-z_{2}\right)\right\} \\
& \leqslant\left\{\left\{\mu\left(z_{1}\right) \vee \mu\left(z_{2}\right)\right\} \vee\left\{\gamma\left(z_{1}\right) \vee \gamma\left(z_{2}\right)\right\}\right\} \\
& =\left\{\mu\left(z_{1}\right) \vee\left\{\mu\left(z_{2}\right) \vee \gamma\left(z_{1}\right)\right\} \vee \gamma\left(z_{2}\right)\right\} \\
& =\left\{\mu\left(z_{1}\right) \vee\left\{\gamma\left(z_{1}\right) \vee \mu\left(z_{2}\right)\right\} \vee \gamma\left(z_{2}\right)\right\} \\
& =\left\{\left\{\mu\left(z_{1}\right) \vee \gamma\left(z_{1}\right)\right\} \vee\left\{\mu\left(z_{2}\right) \vee \gamma\left(z_{2}\right)\right\}\right\} \\
& =\max \left\{(\mu \cup \gamma)\left(z_{1}\right),(\mu \cup \gamma)\left(z_{2}\right)\right\} \\
& =\max \left\{\beta\left(z_{1}\right), \beta\left(z_{2}\right)\right\} \\
& \Rightarrow \beta\left(z_{1}-z_{2}\right) \leqslant \max \left\{\beta\left(z_{1}\right), \beta\left(z_{2}\right)\right\} .
\end{aligned}
$$
Now

Similarly, we have $\beta\left(z_{1} \circ z_{2}\right) \leqslant \max \left\{\beta\left(z_{1}\right), \beta\left(z_{2}\right)\right\}$. Thus $\beta$ is an anti fuzzy LA-sub-ring of an LA-ring R.

$$
\begin{aligned}
\beta\left(z_{1} \circ z_{2}\right)=(\mu \cup \gamma)\left(z_{1} \circ z_{2}\right) & =\max \left\{\mu\left(z_{1} \circ z_{2}\right), \gamma\left(z_{1} \circ z_{2}\right)\right\} \\
& =\max \left\{\mu\left(z_{2} \circ z_{1}\right), \gamma\left(z_{2} \circ z_{1}\right)\right\}=(\mu \cup \gamma)\left(z_{2} \circ z_{1}\right)=\beta\left(z_{2} \circ z_{1}\right) .
\end{aligned}
$$

Hence $\beta=\mu \cup \gamma$ is an anti fuzzy normal LA-sub-ring of R.

Corollary 2.6. If $\left\{\mu_{i}\right\}_{i \in I}$ is a family of anti fuzzy normal $L A$-sub-rings of an $L A$-ring $R$, then $\mu=\cup \mu_{i}$ is also an anti fuzzy normal LA-sub-ring of $\mathrm{R}$.

\section{Direct product of anti fuzzy normal LA-sub-rings}

In this section, we define the direct product of fuzzy subsets $\mu_{1}, \mu_{2}$ of LA-rings $R_{1}, R_{2}$, respectively and investigate the some elementary properties of direct product of anti fuzzy normal LA-sub-rings of an LA-ring $R_{1} \times R_{2}$.

Let $\mu_{1}, \mu_{2}$ is fuzzy subsets of LA-rings $R_{1}, R_{2}$, respectively. The direct product of fuzzy subsets $\mu_{1}, \mu_{2}$ of LA-rings $R_{1}, R_{2}$, is denoted by $\mu_{1} \times \mu_{2}$ and defined by $\left(\mu_{1} \times \mu_{2}\right)\left(x_{1}, x_{2}\right)=\max \left\{\mu_{1}\left(x_{1}\right), \mu_{2}\left(x_{2}\right)\right\}$.

A fuzzy subset $\mu_{1} \times \mu_{2}$ of an LA-ring $R_{1} \times R_{2}$ is said to be an anti fuzzy LA-sub-ring of $R_{1} \times R_{2}$ if

(1) $\left(\mu_{1} \times \mu_{2}\right)(x-y) \leqslant \max \left\{\mu_{1}(x), \mu_{2}(y)\right\} ;$

(2) $\left(\mu_{1} \times \mu_{2}\right)(x y) \leqslant \max \left\{\mu_{1}(x), \mu_{2}(y)\right\}$ for all $x=\left(x_{1}, x_{2}\right), y=\left(y_{1}, y_{2}\right) \in R_{1} \times R_{2}$.

An anti fuzzy LA-sub-ring of an LA-ring $R_{1} \times R_{2}$ is said to be an anti fuzzy normal LA-sub-ring of $R_{1} \times R_{2}$ if $\left(\mu_{1} \times \mu_{2}\right)(x y)=\left(\mu_{1} \times \mu_{2}\right)(y x)$ for all $x=\left(x_{1}, x_{2}\right), y=\left(y_{1}, y_{2}\right) \in R_{1} \times R_{2}$.

Let $A \times B$ is a non-empty subset of an LA-ring $R_{1} \times R_{2}$. The anti characteristic function of $A \times B$ is denoted by $\chi_{A \times B}^{C}$ and defined by

$$
\chi_{A \times B}^{C}: R_{1} \times R_{2} \rightarrow[0,1] \mid x=\left(x_{1}, x_{2}\right) \rightarrow \chi_{A \times B}^{C}(x)= \begin{cases}0, & \text { if } x \in A \times B \\ 1, & \text { if } x \notin A \times B .\end{cases}
$$

Lemma 3.1 ([30, Lemma 4.2]). If $A$ and $B$ are $L A$-sub-rings of LA-rings $R_{1}$ and $R_{2}$, respectively, then $A \times B$ is an LA-sub-ring of an LA-ring $\mathrm{R}_{1} \times \mathrm{R}_{2}$ under the same operations defined as in $\mathrm{R}_{1} \times \mathrm{R}_{2}$. 
Lemma 3.2. Let $A$ and $B$ are $L A$-sub-rings of LA-rings $R_{1}$ and $R_{2}$, respectively. Then $A \times B$ is an LA-sub-ring of an LA-ring $\mathrm{R}_{1} \times \mathrm{R}_{2}$ if and only if the anti characteristic function $\chi_{\mathrm{Z}}^{\mathrm{C}}$ of $\mathrm{Z}=\mathrm{A} \times \mathrm{B}$ is an anti fuzzy normal LA-sub-ring of $\mathrm{R}_{1} \times \mathrm{R}_{2}$.

Proof. Let $Z=A \times B$ is an LA-sub-ring of $R_{1} \times R_{2}$ and $a=\left(a_{1}, a_{2}\right), b=\left(b_{1}, b_{2}\right) \in R_{1} \times R_{2}$. If $a, b \in Z=$ $A \times B$, then by definition of anti characteristic function $\chi_{Z}^{C}(a)=0=\chi_{Z}^{C}(b)$. Since $a-b$ and $a b \in Z, Z$ is an LA-sub-ring of an LA-ring $R_{1} \times R_{2}$. This implies that

$$
\chi_{Z}^{C}(a-b)=0=0 \vee 0=\chi_{Z}^{C}(a) \vee \chi_{Z}^{C}(b) \quad \text { and } \quad \chi_{Z}^{C}(a b)=0=0 \vee 0=\chi_{Z}^{C}(a) \vee \chi_{Z}^{C}(b) .
$$

Thus $\chi_{Z}^{C}(a-b) \leqslant \max \left\{\chi_{Z}^{C}(a), \chi_{Z}^{C}(b)\right\}$ and $\chi_{Z}^{C}(a b) \leqslant \max \left\{\chi_{Z}^{C}(a), \chi_{Z}^{C}(b)\right\}$. Since $a b$ and $b a \in Z$, so $\chi_{Z}^{C}(a b)=$ $0=\chi_{Z}^{C}(b a)$, i.e., $\chi_{Z}^{C}(a b)=\chi_{Z}^{C}(b a)$. Similarly we have

$$
\chi_{Z}^{C}(a-b) \leqslant \max \left\{\chi_{Z}^{C}(a), \chi_{Z}^{C}(b)\right\}, \quad \chi_{Z}^{C}(a b) \leqslant \max \left\{\chi_{Z}^{C}(a), \chi_{Z}^{C}(b)\right\}, \quad \chi_{Z}^{C}(a b)=\chi_{Z}^{C}(b a),
$$

when $a, b \notin Z$. Hence the anti characteristic function $\chi_{Z}^{C}$ of $Z=A \times B$ is an anti fuzzy normal LA-sub-ring of $R_{1} \times R_{2}$.

Conversely, suppose that the anti characteristic function $\chi_{Z}^{C}$ of $Z=A \times B$ is an anti fuzzy normal LA-sub-ring of $R_{1} \times R_{2}$. We have to show that $Z=A \times B$ is an LA-sub-ring of $R_{1} \times R_{2}$. Let $a, b \in Z$, where $a=\left(a_{1}, a_{2}\right)$ and $b=\left(b_{1}, b_{2}\right), a_{1}, b_{1} \in A, a_{2}, b_{2} \in B$, this means that $\chi_{Z}^{C}(a)=0=\chi_{Z}^{C}(b)$. By our supposition

$$
\chi_{Z}^{C}(a-b) \leqslant \chi_{Z}^{C}(a) \vee \chi_{Z}^{C}(b)=0 \vee 0=0 \quad \text { and } \quad \chi_{Z}^{C}(a b) \leqslant \chi_{Z}^{C}(a) \vee \chi_{Z}^{C}(b)=0 \vee 0=0 .
$$

Thus $\chi_{Z}^{C}(a-b)=0=\chi_{Z}^{C}(a b)$, i.e., $a-b$ and $a b \in Z$. Hence $Z=A \times B$ is an LA-sub-ring of $R_{1} \times R_{2}$.

Lemma 3.3. If $\mathrm{X}=\mathrm{A} \times \mathrm{B}$ and $\mathrm{Y}=\mathrm{C} \times \mathrm{D}$ are two LA-sub-rings of an LA-ring $\mathrm{R}_{1} \times \mathrm{R}_{2}$, then their intersection $\mathrm{X} \cap \mathrm{Y}$ is also an LA-sub-ring of $\mathrm{R}_{1} \times \mathrm{R}_{2}$.

Proof. Straight forward.

Theorem 3.4. Let $\mathrm{X}=\mathrm{A} \times \mathrm{B}$ and $\mathrm{Y}=\mathrm{C} \times \mathrm{D}$ are two LA-sub-rings of an LA-ring $\mathrm{R}_{1} \times \mathrm{R}_{2}$. Then $\mathrm{X} \cap \mathrm{Y}$ is an $L A$-sub-ring of $R_{1} \times R_{2}$ if and only if the anti characteristic function $\chi_{Z}^{\mathrm{C}}$ of $\mathrm{Z}=\mathrm{X} \cap \mathrm{Y}$ is an anti fuzzy normal LA-sub-ring of $\mathrm{R}_{1} \times \mathrm{R}_{2}$.

Proof. Let $Z=X \cap Y$ is an LA-sub-ring of an LA-ring $R_{1} \times R_{2}$ and $a=\left(a_{1}, a_{2}\right), b=\left(b_{1}, b_{2}\right) \in R_{1} \times R_{2}$. If $a, b \in Z=X \cap Y$, then by definition of anti characteristic function $\chi_{Z}^{C}(a)=0=\chi_{Z}^{C}(b)$. Since $a-b$ and $a b \in Z, Z$ is an LA-sub-ring of $R_{1} \times R_{2}$. This implies that

$$
\chi_{Z}^{C}(a-b)=0=0 \vee 0=\chi_{Z}^{C}(a) \vee \chi_{Z}^{C}(b) \quad \text { and } \quad \chi_{Z}^{C}(a b)=0=0 \vee 0=\chi_{Z}^{C}(a) \vee \chi_{Z}^{C}(b)
$$

Thus $\chi_{Z}^{C}(a-b) \leqslant \max \left\{\chi_{Z}^{C}(a), \chi_{Z}^{C}(b)\right\}$ and $\chi_{Z}^{C}(a b) \leqslant \max \left\{\chi_{Z}^{C}(a), \chi_{Z}^{C}(b)\right\}$. Since $a b$ and $b a \in Z$, then by definition $\chi_{Z}^{C}(a b)=0=\chi_{Z}^{C}(b a)$, i.e., $\chi_{Z}^{C}(a b)=\chi_{Z}^{C}(b a)$. Similarly we have

$$
\chi_{Z}^{C}(a-b) \leqslant \max \left\{\chi_{Z}^{C}(a), \chi_{Z}^{C}(b)\right\}, \quad \chi_{Z}^{C}(a b) \leqslant \max \left\{\chi_{Z}^{C}(a), \chi_{Z}^{C}(b)\right\}, \quad \chi_{Z}^{C}(a b)=\chi_{Z}^{C}(b a),
$$

when $a, b \notin Z$. Hence the anti characteristic function $\chi_{Z}^{C}$ of $Z$ is an anti fuzzy normal LA-sub-ring of $R_{1} \times R_{2}$.

Conversely, assume that the anti characteristic function $\chi_{Z}^{C}$ of $Z=X \cap Y$ is an anti fuzzy normal LA-sub-ring of an LA-ring $R_{1} \times R_{2}$. Let $a, b \in Z=X \cap Y$, this means that $\chi_{Z}^{C}(a)=0=\chi_{Z}^{C}(b)$. By our assumption

$$
\chi_{Z}^{C}(a-b) \leqslant \chi_{Z}^{C}(a) \vee \chi_{Z}^{C}(b)=0 \vee 0=0 \quad \text { and } \quad \chi_{Z}^{C}(a b) \leqslant \chi_{Z}^{C}(a) \vee \chi_{Z}^{C}(b)=0 \vee 0=0 .
$$

Thus $\chi_{Z}^{C}(a-b)=0=\chi_{Z}^{C}(a b)$, i.e., $a-b$ and $a b \in Z$. Hence $Z$ is an LA-sub-ring of an LA-ring $R_{1} \times R_{2}$. 
Corollary 3.5. Let $\left\{C_{i}\right\}_{i \in I}=\left\{A_{i} \times B_{i}\right\}_{i \in I}$ is a family of LA-sub-rings of an LA-ring $R_{1} \times R_{2}$, then $C=\cap C_{i}$ is an LA-sub-ring of $R_{1} \times R_{2}$ if and only if the anti characteristic function $\chi_{C}^{C}$ of $C=\cap C_{i}$ is an anti fuzzy normal LA-sub-ring of $\mathrm{R}_{1} \times \mathrm{R}_{2}$.

Lemma 3.6. If $\mu$ and $\gamma$ are anti fuzzy normal $L A$-sub-rings of $L A$-rings $R_{1}$ and $R_{2}$, respectively, then $\mu \times \gamma$ is an anti fuzzy normal $L A$-sub-ring of an LA-ring $\mathrm{R}_{1} \times \mathrm{R}_{2}$.

Proof. Let $\mu$ and $\gamma$ are anti fuzzy normal LA-sub-rings of LA-ring $R_{1}$ and $R_{2}$, respectively. We have to show that $\beta=\mu \times \gamma$ is an anti fuzzy normal LA-sub-ring of an LA-ring $R_{1} \times R_{2}$. Let $(a, b),(c, d) \in R_{1} \times R_{2}$. Now

$$
\begin{aligned}
\beta((a, b)-(c, d)) & =(\mu \times \gamma)(a-c, b-d) \\
& =\max \{\mu(a-c), \gamma(b-d)\} \\
& =\mu(a-c) \vee \gamma(b-d) \\
& \leqslant\{\mu(a) \vee \mu(c)\} \vee\{\gamma(b) \vee \gamma(d)\} \\
& =\mu(a) \vee\{\mu(c) \vee \gamma(b)\} \vee \gamma(d) \\
& =\mu(a) \vee\{\gamma(b) \vee \mu(c)\} \vee \gamma(d) \\
& =\{\mu(a) \vee \gamma(b)\} \vee\{\mu(c) \vee \gamma(d)\} \\
& =\max \{(\mu \times \gamma)(a, b),(\mu \times \gamma)(c, d)\} \\
& =\max \{\beta(a, b), \beta(c, d)\} . \\
& \Rightarrow \beta((a, b)-(c, d)) \leqslant \max \{\beta(a, b), \beta(c, d)\} .
\end{aligned}
$$

Similarly, we have $\beta((a, b) \circ(c, d)) \leqslant \max \{\beta(a, b), \beta(c, d)\}$. Thus $\mu \times \gamma$ is an anti fuzzy LA-sub-ring of $R_{1} \times R_{2}$. Now

$$
\begin{aligned}
\beta((a, b) \circ(c, d))=(\mu \times \gamma)(a c, b d) & =\max \{\mu(a c), \gamma(b d)\} \\
& =\max \{\mu(c a), \gamma(d b)\}=(\mu \times \gamma)(c a, d b)=\beta((c, d) \circ(a, b)) .
\end{aligned}
$$

Hence $\mu \times \gamma$ is an anti fuzzy normal LA-sub-ring of $R_{1} \times R_{2}$.

Proposition 3.7. If $\mu=\mu_{1} \times \mu_{2}$ and $\gamma=\gamma_{1} \times \gamma_{2}$ are two anti fuzzy normal $L A$-sub-rings of an LA-ring $R_{1} \times R_{\text {, }}$ then their union $\beta=\mu \cup \gamma$ is also an anti fuzzy normal $L A$-sub-ring of $R_{1} \times R_{2}$.

Proof. Let $\mu=\mu_{1} \times \mu_{2}$ and $\gamma=\gamma_{1} \times \gamma_{2}$ are two anti fuzzy normal LA-sub-rings of an LA-ring $R_{1} \times R_{2}$. We have to show that $\beta=\mu \cup \gamma$ is also an anti fuzzy normal LA-sub-ring of $R_{1} \times R_{2}$. Now

$$
\begin{aligned}
\beta\left(\left(z_{1}, z_{2}\right)-\left(z_{3}, z_{4}\right)\right) & =(\mu \cup \gamma)\left(\left(z_{1}, z_{2}\right)-\left(z_{3}, z_{4}\right)\right) \\
& =\max \left\{\mu\left(\left(z_{1}, z_{2}\right)-\left(z_{3}, z_{4}\right)\right), \gamma\left(\left(z_{1}, z_{2}\right)-\left(z_{3}, z_{4}\right)\right)\right\} \\
& \leqslant\left\{\left\{\mu\left(z_{1}, z_{2}\right) \vee \mu\left(z_{3}, z_{4}\right)\right\} \vee\left\{\gamma\left(z_{1}, z_{2}\right) \vee \gamma\left(z_{3}, z_{4}\right)\right\}\right\} \\
& =\left\{\mu\left(z_{1}, z_{2}\right) \vee\left\{\mu\left(z_{3}, z_{4}\right) \vee \gamma\left(z_{1}, z_{2}\right)\right\} \vee \gamma\left(z_{3}, z_{4}\right)\right\} \\
& =\left\{\mu\left(z_{1}, z_{2}\right) \vee\left\{\gamma\left(z_{1}, z_{2}\right) \vee \mu\left(z_{3}, z_{4}\right)\right\} \vee \gamma\left(z_{3}, z_{4}\right)\right\} \\
& =\left\{\left\{\mu\left(z_{1}, z_{2}\right) \vee \gamma\left(z_{1}, z_{2}\right)\right\} \vee\left\{\mu\left(z_{3}, z_{4}\right) \vee \gamma\left(z_{3}, z_{4}\right)\right\}\right\} \\
& =\max \left\{(\mu \cup \gamma)\left(z_{1}, z_{2}\right),(\mu \cup \gamma)\left(z_{3}, z_{4}\right)\right\} \\
& =\max \left\{\beta\left(z_{1}, z_{2}\right), \beta\left(z_{3}, z_{4}\right)\right\} \\
& \Rightarrow \beta\left(\left(z_{1}, z_{2}\right)-\left(z_{3}, z_{4}\right)\right) \leqslant \max \left\{\beta\left(z_{1}, z_{2}\right), \beta\left(z_{3}, z_{4}\right)\right\} .
\end{aligned}
$$

Similarly, we have $\beta\left(\left(z_{1}, z_{2}\right) \circ\left(z_{3}, z_{4}\right)\right) \leqslant \max \left\{\beta\left(z_{1}, z_{2}\right), \beta\left(z_{3}, z_{4}\right)\right\}$. Thus $\beta=\mu \cup \gamma$ is an anti fuzzy LA-subring of an LA-ring $R_{1} \times R_{2}$. Now

$$
\beta\left(\left(z_{1}, z_{2}\right) \circ\left(z_{3}, z_{4}\right)\right)=(\mu \cup \gamma)\left(\left(z_{1}, z_{2}\right) \circ\left(z_{3}, z_{4}\right)\right)=\max \left\{\mu\left(\left(z_{1}, z_{2}\right) \circ\left(z_{3}, z_{4}\right)\right), \gamma\left(\left(z_{1}, z_{2}\right) \circ\left(z_{3}, z_{4}\right)\right)\right\}
$$




$$
\begin{aligned}
& =\max \left\{\mu\left(\left(z_{3}, z_{4}\right) \circ\left(z_{1}, z_{2}\right)\right), \gamma\left(\left(z_{3}, z_{4}\right) \circ\left(z_{1}, z_{2}\right)\right)\right\} \\
& =(\mu \cup \gamma)\left(\left(z_{3}, z_{4}\right) \circ\left(z_{1}, z_{2}\right)\right)=\beta\left(\left(z_{3}, z_{4}\right) \circ\left(z_{1}, z_{2}\right)\right) .
\end{aligned}
$$

Hence $\beta=\mu \cup \gamma$ is an anti fuzzy normal LA-sub-ring of an LA-ring $R_{1} \times R_{2}$.

Corollary 3.8. If $\left\{\beta_{i}\right\}_{i \in I}=\left\{\mu_{i} \times \gamma_{i}\right\}_{i \in I}$ is a family of anti fuzzy normal LA-sub-rings of an LA-ring $R_{1} \times R_{2}$, then $\beta=\cup \beta_{i}$ is also an anti fuzzy normal $L A$-sub-ring of $R_{1} \times R_{2}$.

Theorem 3.9. If $\mu=\mu_{1} \times \mu_{2}$ and $\gamma=\gamma_{1} \times \gamma_{2}$ are anti fuzzy normal LA-sub-rings of LA-rings $R^{\prime}=R_{1} \times R_{2}$ and $R^{\prime \prime}=R_{3} \times R_{4}$, respectively, then $\beta=\mu \times \gamma$ is an anti fuzzy normal LA-sub-ring of an LA-ring $R^{\prime} \times R^{\prime \prime}=$ $\left(R_{1} \times R_{2}\right) \times\left(R_{3} \times R_{4}\right)$.

Proof. Let $\mu=\mu_{1} \times \mu_{2}$ and $\gamma=\gamma_{1} \times \gamma_{2}$ are anti fuzzy normal LA-sub-rings of LA-rings $R^{\prime}=R_{1} \times R_{2}$ and $R^{\prime \prime}=R_{3} \times R_{4}$, respectively. We have to show that $\beta=\mu \times \gamma$ is an anti fuzzy normal LA-sub-ring of an LA-ring $R^{\prime} \times R^{\prime \prime}$. Now

$$
\begin{aligned}
\beta\left(\left(\left(z_{1}, z_{2}\right),\left(z_{3}, z_{4}\right)\right)-\left(\left(z_{5}, z_{6}\right),\left(z_{7}, z_{8}\right)\right)\right) & =(\mu \times \gamma)\left(\left(\left(z_{1}, z_{2}\right),\left(z_{3}, z_{4}\right)\right)-\left(\left(z_{5}, z_{6}\right),\left(z_{7}, z_{8}\right)\right)\right) \\
& =(\mu \times \gamma)\left(\left(\left(z_{1}, z_{2}\right)-\left(z_{5}, z_{6}\right)\right),\left(\left(z_{3}, z_{4}\right)-\left(z_{7}, z_{8}\right)\right)\right) \\
& =\max \left\{\mu\left(\left(z_{1}, z_{2}\right)-\left(z_{5}, z_{6}\right)\right), \gamma\left(\left(z_{3}, z_{4}\right)-\left(z_{7}, z_{8}\right)\right)\right\} \\
& \leqslant \max \left\{\left(\mu\left(z_{1}, z_{2}\right) \vee \mu\left(z_{5}, z_{6}\right)\right),\left(\gamma\left(z_{3}, z_{4}\right) \vee \gamma\left(z_{7}, z_{8}\right)\right)\right\} \\
& =\left\{\left(\mu\left(z_{1}, z_{2}\right) \vee \mu\left(z_{5}, z_{6}\right)\right) \vee\left(\gamma\left(z_{3}, z_{4}\right) \vee \gamma\left(z_{7}, z_{8}\right)\right)\right\} \\
& =\left\{\left(\mu\left(z_{1}, z_{2}\right) \vee \gamma\left(z_{3}, z_{4}\right)\right) \vee\left(\mu\left(z_{5}, z_{6}\right) \vee \gamma\left(z_{7}, z_{8}\right)\right)\right\} \\
& =\max \left\{\left(\mu\left(z_{1}, z_{2}\right) \vee \gamma\left(z_{3}, z_{4}\right)\right),\left(\mu\left(z_{5}, z_{6}\right) \vee \gamma\left(z_{7}, z_{8}\right)\right)\right\} \\
& =\max \left\{(\mu \times \gamma)\left(\left(z_{1}, z_{2}\right),\left(z_{3}, z_{4}\right)\right),(\mu \times \gamma)\left(\left(z_{5}, z_{6}\right),\left(z_{7}, z_{8}\right)\right)\right\} \\
& =\max \left\{\beta\left(\left(z_{1}, z_{2}\right),\left(z_{3}, z_{4}\right)\right), \beta\left(\left(z_{5}, z_{6}\right),\left(z_{7}, z_{8}\right)\right)\right\} .
\end{aligned}
$$

Similarly, we have

$$
\beta\left(\left(\left(z_{1}, z_{2}\right),\left(z_{3}, z_{4}\right)\right) \circ\left(\left(z_{5}, z_{6}\right),\left(z_{7}, z_{8}\right)\right)\right) \leqslant \max \left\{\beta\left(\left(z_{1}, z_{2}\right),\left(z_{3}, z_{4}\right)\right), \beta\left(\left(z_{5}, z_{6}\right),\left(z_{7}, z_{8}\right)\right)\right\} .
$$

Thus $\beta=\mu \times \gamma$ is an anti fuzzy LA-sub-ring of an LA-ring $R \prime \times R^{\prime \prime}$. Now

$$
\begin{aligned}
\beta\left(\left(\left(z_{1}, z_{2}\right),\left(z_{3}, z_{4}\right)\right) \circ\left(\left(z_{5}, z_{6}\right),\left(z_{7}, z_{8}\right)\right)\right) & =(\mu \times \gamma)\left(\left(\left(z_{1}, z_{2}\right),\left(z_{3}, z_{4}\right)\right) \circ\left(\left(z_{5}, z_{6}\right),\left(z_{7}, z_{8}\right)\right)\right) \\
& =(\mu \times \gamma)\left(\left(\left(z_{1}, z_{2}\right) \circ\left(z_{5}, z_{6}\right)\right),\left(\left(z_{3}, z_{4}\right) \circ\left(z_{7}, z_{8}\right)\right)\right) \\
& =\max \left\{\mu\left(\left(z_{1}, z_{2}\right) \circ\left(z_{5}, z_{6}\right)\right), \gamma\left(\left(z_{3}, z_{4}\right) \circ\left(z_{7}, z_{8}\right)\right)\right\} \\
& =\max \left\{\mu\left(\left(z_{5}, z_{6}\right) \circ\left(z_{1}, z_{2}\right)\right), \gamma\left(\left(z_{7}, z_{8}\right) \circ\left(z_{3}, z_{4}\right)\right)\right\} \\
& =(\mu \times \gamma)\left(\left(\left(z_{5}, z_{6}\right) \circ\left(z_{1}, z_{2}\right)\right),\left(\left(z_{7}, z_{8}\right) \circ\left(z_{3}, z_{4}\right)\right)\right) \\
& =(\mu \times \gamma)\left(\left(\left(z_{5}, z_{6}\right),\left(z_{7}, z_{8}\right)\right) \circ\left(\left(z_{1}, z_{2}\right),\left(z_{3}, z_{4}\right)\right)\right) \\
& =\beta\left(\left(\left(z_{5}, z_{6}\right),\left(z_{7}, z_{8}\right)\right) \circ\left(\left(z_{1}, z_{2}\right),\left(z_{3}, z_{4}\right)\right)\right) .
\end{aligned}
$$

Hence $\beta=\mu \times \gamma$ is an anti fuzzy normal LA-sub-ring of an LA-ring $R \prime \times R^{\prime \prime}$.

Lemma 3.10. Let $\mu$ and $\gamma$ are fuzzy subsets of $L A$-rings $R_{1}$ and $R_{2}$ with left identities $e_{1}$ and $e_{2}$, respectively. If $\mu \times \gamma$ is an anti fuzzy $L A$-sub-ring of an LA-ring $R_{1} \times R_{2}$, then at least one of the following two statements must hold.

1. $\mu(x) \geqslant \gamma\left(e_{2}\right)$, for all $x \in R_{1}$;

2. $\mu(x) \geqslant \gamma\left(e_{1}\right)$, for all $x \in R_{2}$. 
Proof. Let $\mu \times \gamma$ is an anti fuzzy LA-sub-ring of $R_{1} \times R_{2}$. By contra-position, suppose that none of the statements 1 and 2 holds. Then we can find $a$ and $b$ in $R_{1}$ and $R_{2}$, respectively such that

$$
\mu(a) \leqslant \gamma\left(e_{2}\right) \text { and } \mu(b) \leqslant \gamma\left(e_{1}\right) .
$$

Thus we have

$$
(\mu \times \gamma)(a, b)=\max \{\mu(a), \gamma(b)\} \leqslant \max \left\{\mu\left(e_{1}\right), \gamma\left(e_{2}\right)\right\}=(\mu \times \gamma)\left(e_{1}, e_{2}\right) .
$$

Therefore $\mu \times \gamma$ is not an anti fuzzy LA-sub-ring of $R_{1} \times R_{2}$. Hence either $\mu(x) \geqslant \gamma\left(e_{2}\right)$ for all $x \in R_{1}$ or $\mu(x) \geqslant \gamma\left(e_{1}\right)$ for all $x \in R_{2}$.

Lemma 3.11. Let $\mu$ and $\gamma$ are fuzzy subsets of $L A$-rings $R_{1}$ and $R_{2}$ with left identities $e_{1}$ and $e_{2}$, respectively and $\mu \times \gamma$ is an anti fuzzy normal LA-sub-ring of an LA-ring $R_{1} \times R_{2}$. Then the following conditions are true.

1. If $\mu(x) \geqslant \gamma\left(e_{2}\right)$, for all $x \in R_{1}$, then $\mu$ is an anti fuzzy normal $L A$-sub-ring of $R_{1}$.

2. If $\mu(x) \geqslant \gamma\left(e_{1}\right)$, for all $x \in R_{2}$, then $\gamma$ is an anti fuzzy normal $L A$-sub-ring of $R_{2}$.

Proof. 1. Let $\mu(x) \geqslant \gamma\left(e_{2}\right)$ for all $x \in R_{1}$, and $y \in R_{1}$. We have to show that $\mu$ is an anti fuzzy normal LA-sub-ring of $R_{1}$. Now

$$
\begin{aligned}
\mu(x-y) & =\mu(x+(-y)) \\
& =\max \left\{\mu(x+(-y)), \gamma\left(e_{2}+\left(-e_{2}\right)\right)\right\} \\
& =(\mu \times \gamma)\left(x+(-y), e_{2}+\left(-e_{2}\right)\right) \\
& =(\mu \times \gamma)\left(\left(x, e_{2}\right)+\left(-y,-e_{2}\right)\right) \\
& =(\mu \times \gamma)\left(\left(x, e_{2}\right)-\left(y, e_{2}\right)\right) \\
& \leqslant(\mu \times \gamma)\left(x, e_{2}\right) \vee(\mu \times \gamma)\left(y, e_{2}\right) \\
& =\max \left\{\max \left\{\mu(x), \gamma\left(e_{2}\right)\right\}, \max \left\{\mu(y), \gamma\left(e_{2}\right)\right\}\right\} \\
& =\mu(x) \vee \mu(y),
\end{aligned}
$$

and

$$
\begin{aligned}
\mu(x y) & =\max \left\{\mu(x y), \gamma\left(e_{2} e_{2}\right)\right\} \\
& =(\mu \times \gamma)\left(x y, e_{2} e_{2}\right) \\
& =(\mu \times \gamma)\left(\left(x, e_{2}\right) \circ\left(y, e_{2}\right)\right) \\
& \leqslant(\mu \times \gamma)\left(x, e_{2}\right) \vee \mu \times \gamma\left(y, e_{2}\right) \\
& =\max \left\{\max \left\{\mu(x), \gamma\left(e_{2}\right)\right\}, \max \left\{\mu(y), \gamma\left(e_{2}\right)\right\}\right\} \\
& =\mu(x) \vee \mu(y) .
\end{aligned}
$$

Thus $\mu$ is an anti fuzzy LA-sub-ring of $R_{1}$. Now

$$
\begin{aligned}
\mu(x y) & =\max \left\{\mu(x y), \gamma\left(e_{2} e_{2}\right)\right\} \\
& =(\mu \times \gamma)\left(x y, e_{2} e_{2}\right) \\
& =(\mu \times \gamma)\left(\left(x, e_{2}\right) \circ\left(y, e_{2}\right)\right) \\
& =(\mu \times \gamma)\left(\left(y, e_{2}\right) \circ\left(x, e_{2}\right)\right)=(\mu \times \gamma)\left(y x, e_{2} e_{2}\right)=\max \left\{\mu(y x), \gamma\left(e_{2} e_{2}\right)\right\}=\mu(y x) .
\end{aligned}
$$

Hence $\mu$ is an anti fuzzy normal LA-sub-ring of $R_{1} .2$ is same as 1 . 


\section{Direct product of finite anti fuzzy normal LA-sub-rings}

In this section, we define the direct product of fuzzy subsets $\mu_{1}, \mu_{2}, \ldots, \mu_{n}$ of LA-rings $R_{1}, R_{2}, \ldots, R_{n}$, respectively and examine the some fundamental properties of direct product of anti fuzzy normal LAsub-rings of an LA-ring $R_{1} \times R_{2} \times \cdots \times R_{n}$.

Let $\mu_{1}, \mu_{2}, \ldots, \mu_{n}$ are fuzzy subsets of LA-rings $R_{1}, R_{2}, \ldots, R_{n}$, respectively. The direct of fuzzy subsets $\mu_{1}, \mu_{2}, \ldots, \mu_{n}$, is denoted by $\mu_{1} \times \mu_{2} \times \cdots \times \mu_{n}$ and defined by $\left(\mu_{1} \times \mu_{2} \times \cdots \times \mu_{n}\right)\left(x_{1}, x_{2}, \ldots, x_{n}\right)=$ $\max \left\{\mu_{1}\left(x_{1}\right), \mu_{2}\left(x_{2}\right), \ldots, \mu_{n}\left(x_{n}\right)\right\}$.

A fuzzy subset $\mu_{1} \times \mu_{2} \times \cdots \times \mu_{n}$ of an LA-ring $R_{1} \times R_{2} \times \cdots \times R_{n}$ is said to be an anti fuzzy LA-subring of $R_{1} \times R_{2} \times \cdots \times R_{n}$ if

1. $\left(\mu_{1} \times \mu_{2} \times \cdots \times \mu_{n}\right)(x-y) \leqslant \max \left\{\left(\mu_{1} \times \mu_{2} \times \cdots \times \mu_{n}\right)(x),\left(\mu_{1} \times \mu_{2} \times \cdots \times \mu_{n}\right)(y)\right\} ;$

2. $\left(\mu_{1} \times \mu_{2} \times \cdots \times \mu_{n}\right)(x y) \leqslant \max \left\{\left(\mu_{1} \times \mu_{2} \times \cdots \times \mu_{n}\right)(x),\left(\mu_{1} \times \mu_{2} \times \cdots \times \mu_{n}\right)(y)\right\}$ for all $x=$ $\left(x_{1}, x_{2}, \ldots, x_{n}\right), y=\left(y_{1}, y_{2}, \ldots, y_{n}\right) \in R_{1} \times R_{2} \times \cdots \times R_{n}$.

An anti fuzzy LA-sub-ring of an LA-ring $R_{1} \times R_{2} \times \cdots \times R_{n}$ is said to be an anti fuzzy normal LA-subring of $R_{1} \times R_{2} \times \cdots \times R_{n}$ if $\left(\mu_{1} \times \mu_{2} \times \cdots \times \mu_{n}\right)(x y)=\left(\mu_{1} \times \mu_{2} \times \cdots \times \mu_{n}\right)(y x)$ for all $x=\left(x_{1}, x_{2}, \ldots, x_{n}\right)$, $y=\left(y_{1}, y_{2}, \ldots, y_{n}\right) \in R_{1} \times R_{2} \times \cdots \times R_{n}$.

Let $A_{1} \times A_{2} \times \cdots \times A_{n}$ is a non-empty subset of an LA-ring $R=R_{1} \times R_{2} \times \cdots \times R_{n}$. The anti characteristic function of $A=A_{1} \times A_{2} \times \cdots \times A_{n}$ is denoted by $\chi_{A}^{C}$ and defined by

$$
\chi_{\AA}^{C}: R \rightarrow[0,1] \mid x=\left(x_{1}, x_{2}, \ldots, x_{n}\right) \rightarrow \chi_{\AA}^{C}(x)= \begin{cases}0, & \text { if } x \in A, \\ 1, & \text { if } x \notin A .\end{cases}
$$

Lemma 4.1. If $A_{1}, A_{2}, \ldots, A_{n}$ are LA-sub-rings of LA-rings $R_{1}, R_{2}, \ldots, R_{n}$, respectively, then $A_{1} \times A_{2} \times \cdots \times A_{n}$ is an LA-sub-ring of an LA-ring $\mathrm{R}_{1} \times \mathrm{R}_{2} \times \cdots \times \mathrm{R}_{\mathrm{n}}$ under the same operations defined as in [30].

Proof. Straight forward.

Proposition 4.2. Let $A_{1}, A_{2}, \ldots, A_{n}$ are LA-sub-rings of LA-rings $R_{1}, R_{2}, \ldots, R_{n}$, respectively. Then $A_{1} \times A_{2} \times$ $\cdots \times A_{n}$ is an LA-sub-ring of an LA-ring $R_{1} \times R_{2} \times \cdots \times R_{n}$ if and only if the anti characteristic function $\chi_{A}^{C}$ of $A=A_{1} \times A_{2} \times \cdots \times A_{n}$ is an anti fuzzy normal LA-sub-ring of $R_{1} \times R_{2} \times \cdots \times R_{n}$.

Proof. Let $A=A_{1} \times A_{2} \times \cdots \times A_{n}$ is an LA-sub-ring of $R_{1} \times R_{2} \times \cdots \times R_{n}$ and $a=\left(a_{1}, a_{2}, \ldots, a_{n}\right), b=$ $\left(b_{1}, b_{2}, \ldots, b_{n}\right) \in R_{1} \times R_{2} \times \cdots \times R_{n}$. If $a, b \in A=A_{1} \times A_{2} \times \cdots \times A_{n}$, then by definition of anti characteristic function $\chi_{A}^{C}(a)=0=\chi_{\AA}^{C}(b)$. Since $a-b$ and $a b \in A$, $A$ is an LA-sub-ring of $R_{1} \times R_{2} \times \cdots \times R_{n}$. This implies that

$$
\chi_{\AA}^{C}(a-b)=0=0 \vee 0=\chi_{\AA}^{C}(a) \vee \chi_{\AA}^{C}(b) \quad \text { and } \quad \chi_{\AA}^{C}(a b)=0=0 \vee 0=\chi_{\AA}^{C}(a) \vee \chi_{\AA}^{C}(b) .
$$

Thus $\chi_{\AA}^{C}(a-b) \leqslant \max \left\{\chi_{\AA}^{C}(a), \chi_{\AA}^{C}(b)\right\}$ and $\chi_{\AA}^{C}(a b) \leqslant \max \left\{\chi_{\AA}^{C}(a), \chi_{\AA}^{C}(b)\right\}$. Since $a b$ and ba $\in A$, so $\chi_{\AA}^{C}(a b)=0=\chi_{\AA}^{C}(b a)$, i.e., $\chi_{\AA}^{C}(a b)=\chi_{A}^{C}(b a)$. Similarly, we have

$$
\chi_{\AA}^{C}(a-b) \leqslant \max \left\{\chi_{\AA}^{C}(a), \chi_{\AA}^{C}(b)\right\}, \quad \chi_{\AA}^{C}(a b) \leqslant \max \left\{\chi_{\AA}^{C}(a), \chi_{\AA}^{C}(b)\right\}, \quad \chi_{\AA}^{C}(a b)=\chi_{\AA}^{C}(b a),
$$

when $a, b \notin A$. Hence the anti characteristic function $\chi_{\AA}^{C}$ of $A=A_{1} \times A_{2} \times \cdots \times A_{n}$ is an anti fuzzy normal LA-sub-ring of $R_{1} \times R_{2} \times \cdots \times R_{n}$.

Conversely, assume that the anti characteristic function $\chi_{\AA}^{C}$ of $A=A_{1} \times A_{2} \times \cdots \times A_{n}$ is an anti fuzzy normal LA-sub-ring of $R_{1} \times R_{2} \times \cdots \times R_{n}$. We have to show that $A=A_{1} \times A_{2} \times \cdots \times A_{n}$ is an LA-sub-ring of $R_{1} \times R_{2} \times \cdots \times R_{n}$. Let $a, b \in A$, where $a=\left(a_{1}, a, \ldots, a_{n}\right)$ and $b=\left(b_{1}, b_{2}, \ldots, b_{n}\right)$, this means that $\chi_{\AA}^{C}(a)=0=\chi_{\AA}^{C}(b)$. By our supposition

$$
\chi_{\AA}^{C}(a-b) \leqslant \chi_{\AA}^{C}(a) \vee \chi_{\AA}^{C}(b)=0 \vee 0=0 \quad \text { and } \quad \chi_{\AA}^{C}(a b) \leqslant \chi_{\AA}^{C}(a) \vee \chi_{\AA}^{C}(b)=0 \vee 0=0 .
$$

Thus $\chi_{\AA}^{C}(a-b)=0=\chi_{\AA}^{C}(a b)$, i.e., $a-b$ and $a b \in A$. Hence $A=A_{1} \times A_{2} \times \cdots \times A_{n}$ is an LA-sub-ring of an LA-ring $R_{1} \times R_{2} \times \cdots \times R_{n}$. 
Lemma 4.3. If $A=A_{1} \times A_{2} \times \cdots \times A_{n}$ and $B=B_{1} \times B_{2} \times \cdots \times B_{n}$ are two LA-sub-rings of an LA-ring $R_{1} \times R_{2} \times \cdots \times R_{n}$, then their intersection $A \cap B$ is also an LA-sub-ring of $R_{1} \times R_{2} \times \cdots \times R_{n}$.

Proof. Straight forward.

Theorem 4.4. Let $\mathrm{A}=\mathrm{A}_{1} \times \mathrm{A}_{2} \times \cdots \times \mathrm{A}_{n}$ and $\mathrm{B}=\mathrm{B}_{1} \times \mathrm{B}_{2} \times \cdots \times \mathrm{B}_{n}$ are two LA-sub-rings of an LA-ring $R_{1} \times R_{2} \times \cdots \times R_{n}$. Then $A \cap B$ is an LA-sub-ring of $R_{1} \times R_{2} \times \cdots \times R_{n}$ if and only if the anti characteristic function $\chi_{Z}^{C}$ of $Z=A \cap B$ is an anti fuzzy normal LA-sub-ring of $R_{1} \times R_{2} \times \cdots \times R_{n}$.

Proof. Let $Z=A \cap B$ is an LA-sub-ring of $R_{1} \times R_{2} \times \cdots \times R_{n}$ and $a=\left(a_{1}, a_{2}, \ldots, a_{n}\right), b=\left(b_{1}, b_{1}, \ldots, b_{n}\right) \in$ $R_{1} \times R_{2} \times \cdots \times R_{n}$. If $a, b \in Z=A \cap B$, then by definition of anti characteristic function $\chi_{Z}^{C}(a)=0=\chi_{Z}^{C}(b)$. Since $a-b$ and $a b \in Z$. This implies that

$$
\chi_{Z}^{C}(a-b)=0=0 \vee 0=\chi_{Z}^{C}(a) \vee \chi_{Z}^{C}(b) \quad \text { and } \quad \chi_{Z}^{C}(a b)=0=0 \vee 0=\chi_{Z}^{C}(a) \vee \chi_{Z}^{C}(b)
$$

Thus $\chi_{Z}^{C}(a-b) \leqslant \max \left\{\chi_{Z}^{C}(a), \chi_{Z}^{C}(b)\right\}$ and $\chi_{Z}^{C}(a b) \leqslant \max \left\{\chi_{Z}^{C}(a), \chi_{Z}^{C}(b)\right\}$. As $a b$ and $b a \in Z$, by definition $\chi_{Z}^{C}(a b)=0=\chi_{Z}^{C}(b a)$, i.e., $\chi_{Z}^{C}(a b)=\chi_{Z}^{C}(b a)$. Similarly, we have

$$
\chi_{Z}^{C}(a-b) \leqslant \max \left\{\chi_{Z}^{C}(a), \chi_{Z}^{C}(b)\right\}, \quad \chi_{Z}^{C}(a b) \leqslant \max \left\{\chi_{Z}^{C}(a), \chi_{Z}^{C}(b)\right\}, \quad \chi_{Z}^{C}(a b)=\chi_{Z}^{C}(b a),
$$

when $a, b \notin Z$. Hence the anti characteristic function $\chi_{Z}^{C}$ of $Z$ is an anti fuzzy normal LA-sub-ring of $\mathrm{R}_{1} \times \mathrm{R}_{2} \times \cdots \times \mathrm{R}_{\mathrm{n}}$.

Conversely, assume that the anti characteristic function $\chi_{Z}^{C}$ of $Z=A \cap B$ is an anti fuzzy normal LA-sub-ring of $R_{1} \times R_{2} \times \cdots \times R_{n}$. Let $a, b \in Z=A \cap B$, this means that $\chi_{Z}^{C}(a)=0=\chi_{Z}^{C}(b)$. By our supposition

$$
\chi_{Z}^{C}(a-b) \leqslant \chi_{Z}^{C}(a) \vee \chi_{Z}^{C}(b)=0 \vee 0=0 \quad \text { and } \quad \chi_{Z}^{C}(a b) \leqslant \chi_{Z}^{C}(a) \vee \chi_{Z}^{C}(b)=0 \vee 0=0 .
$$

Thus $\chi_{Z}^{C}(a-b)=0=\chi_{Z}^{C}(a b)$, i.e., $a-b$ and $a b \in Z$. Hence $Z$ is an LA-sub-ring of $R_{1} \times R_{2} \times \cdots \times R_{n}$.

Corollary 4.5. Let $\left\{A_{i}\right\}_{i \in I}=\left\{A_{i 1} \times A_{i 2} \times \cdots \times A_{i n}\right\}_{i \in I}$ is a family of LA-sub-rings of an LA-ring $R_{1} \times R_{2} \times$ $\cdots \times R_{n}$, then $A=\cap A_{i}$ is an LA-sub-ring of $R_{1} \times R_{2} \times \cdots \times R_{n}$ if and only if the anti characteristic function $\chi_{\AA}^{C}$ of $A=\cap A_{i}$ is an anti fuzzy normal LA-sub-ring of $R_{1} \times R_{2} \times \cdots \times R_{n}$.

Theorem 4.6. If $\mu=\mu_{1} \times \mu_{2} \times \cdots \times \mu_{\mathrm{n}}$ and $\gamma=\gamma_{1} \times \gamma_{2} \times \cdots \times \gamma_{\mathrm{n}}$ are two anti fuzzy normal LA-sub-rings of an LA-ring $R_{1} \times R_{2} \times \cdots \times R_{n}$, then their union $\beta=\mu \cup \gamma$ is also an anti fuzzy normal LA-sub-ring of $\mathrm{R}_{1} \times \mathrm{R}_{2} \times \cdots \times \mathrm{R}_{\mathrm{n}}$.

Proof. Let $\mu=\mu_{1} \times \mu_{2} \times \cdots \times \mu_{n}$ and $\gamma=\gamma_{1} \times \gamma_{2} \times \cdots \times \gamma_{n}$ are two anti fuzzy normal LA-sub-rings of an LA-ring $R_{1} \times R_{2} \times \cdots \times R_{n}$. We have to show that $\beta=\mu \cup \gamma$ is also an anti fuzzy normal LA-sub-ring of $R_{1} \times R_{2} \times \cdots \times R_{n}$. Let $z=\left(z_{1}, z_{2}, \ldots, z_{n}\right)$ and $w=\left(w_{1}, w_{2}, \ldots, w_{n}\right) \in R_{1} \times R_{2} \times \cdots \times R_{n}$. Now

$$
\begin{aligned}
\beta(z-w) & =(\mu \cup \gamma)(z-w)=\max \{\mu(z-w), \gamma(z-w)\} \\
& \leqslant\{\{\mu(z) \vee \mu(w)\} \vee\{\gamma(z) \vee \gamma(w)\}\} \\
& =\{\mu(z) \vee\{\mu(w) \vee \gamma(z)\} \vee \gamma(w)\} \\
& =\{\mu(z) \vee\{\gamma(z) \vee \mu(w)\} \vee \gamma(w)\} \\
& =\{\{\mu(z) \vee \gamma(z)\} \vee\{\mu(w) \vee \gamma(w)\}\} \\
& =\max \{(\mu \cup \gamma)(z),(\mu \cup \gamma)(w)\} \\
& =\max \{\beta(z), \beta(w)\}
\end{aligned}
$$

Thus $\beta\left(\left(z_{1}, z_{2}, \ldots, z_{n}\right)-\left(w_{1}, w_{2}, \ldots, w_{n}\right)\right) \leqslant \max \left\{\beta\left(z_{1}, z_{2}, \ldots, z_{n}\right), \beta\left(w_{1}, w_{2}, \ldots, w_{n}\right)\right\}$. Similarly, we have

$$
\beta\left(\left(z_{1}, z_{2}, \ldots, z_{n}\right) \circ\left(w_{1}, w_{2}, \ldots, w_{n}\right)\right) \leqslant \max \left\{\beta\left(z_{1}, z_{2}, \ldots, z_{n}\right), \beta\left(w_{1}, w_{2}, \ldots, w_{n}\right)\right\} .
$$


Thus $\beta=\mu \cup \gamma$ is an anti fuzzy LA-sub-ring of an LA-ring $R_{1} \times R_{2} \times \cdots \times R_{n}$. Now

$$
\begin{aligned}
\beta\left(\left(z_{1}, z_{2}, \ldots, z_{n}\right) \circ\left(w_{1}, w_{2}, \ldots, w_{n}\right)\right) & =(\mu \cup \gamma)\left(z_{1} w_{1}, z_{2} w_{2}, \ldots, z_{n} w_{n}\right) \\
& =\max \left\{\mu\left(z_{1} w_{1}, z_{2} w_{2}, \ldots, z_{n} w_{n}\right), \gamma\left(z_{1} w_{1}, z_{2} w_{2}, \ldots, z_{n} w_{n}\right)\right\} \\
& =\max \left\{\mu\left(w_{1} z_{1}, w_{2} z_{2}, \ldots, w_{n} z_{n}\right), \gamma\left(w_{1} z_{1}, w_{2} z_{2}, \ldots, w_{n} z_{n}\right)\right\} \\
& =(\mu \cup \gamma)\left(w_{1} z_{1}, w_{2} z_{2}, \ldots, w_{n} z_{n}\right) \\
& =\beta\left(\left(w_{1}, w_{2}, \ldots, w_{n}\right) \circ\left(z_{1}, z_{2}, \ldots, z_{n}\right)\right) .
\end{aligned}
$$

Hence $\beta=\mu \cup \gamma$ is an anti fuzzy normal LA-sub-ring of an LA-ring $R_{1} \times R_{2} \times \cdots \times R_{n}$.

Corollary 4.7. If $\left\{\mu_{i}\right\}_{i \in I}=\left\{\mu_{i 1} \times \mu_{i 2} \times \cdots \times \mu_{i n}\right\}_{i \in \mathrm{I}}$ is a family of anti fuzzy normal LA-sub-rings of an LA-ring $\mathrm{R}_{1} \times \mathrm{R}_{2} \times \cdots \times \mathrm{R}_{\mathrm{n}}$, then $\mu=\cup \mu_{\mathrm{i}}$ is also an anti fuzzy normal LA-sub-ring of $\mathrm{R}_{1} \times \mathrm{R}_{2} \times \cdots \times \mathrm{R}_{\mathrm{n}}$.

Proposition 4.8. Let $\mu=\mu_{1} \times \mu_{2} \times \cdots \times \mu_{n}$ and $\gamma=\gamma_{1} \times \gamma_{2} \times \cdots \times \gamma_{n}$ are fuzzy subsets of LA-rings $\mathrm{R}=\mathrm{R}_{1} \times \mathrm{R}_{2} \times \cdots \times \mathrm{R}_{\mathrm{n}}$ and $\mathrm{R}^{\prime}=\mathrm{R}_{1}^{\prime} \times \mathrm{R}_{2}^{\prime} \times \cdots \times \mathrm{R}_{\mathrm{n}}^{\prime}$ with left identities $\mathrm{e}=\left(\mathrm{e}_{1}, \mathrm{e}_{2}, \ldots, \mathrm{e}_{\mathrm{n}}\right)$ and $\mathrm{e}^{\prime}=$ $\left(e_{1}^{\prime}, e_{2} \prime, \ldots, e_{n}^{\prime}\right)$, respectively. If $\mu \times \gamma$ is an anti fuzzy LA-sub-ring of an LA-ring $\mathrm{R} \times \mathrm{R}^{\prime}$, then at least one of the following two statements must hold.

1. $\mu(x) \geqslant \gamma\left(e^{\prime}\right)$ for all $x \in R$;

2. $\mu(x) \geqslant \gamma(e)$ for all $x \in R^{\prime}$.

Proof. Let $\mu \times \gamma$ is an anti fuzzy LA-sub-ring of $R \times R^{\prime}$. By contraposition, suppose that none of the statements 1 and 2 holds. Then we can find $a$ and $b$ in $R$ and $R^{\prime}$, respectively such that

$$
\mu(a) \leqslant \gamma\left(e^{\prime}\right) \text { and } \mu(b) \leqslant \gamma(e) .
$$

Thus, we have

$$
(\mu \times \gamma)(a, b)=\max \{\mu(a), \gamma(b)\} \leqslant \max \left\{\mu(e), \gamma\left(e^{\prime}\right)\right\}=(\mu \times \gamma)(e, e) .
$$

Therefore $\mu \times \gamma$ is not an anti fuzzy LA-sub-ring of $R \times R^{\prime}$. Hence either $\mu(x) \geqslant \gamma\left(e^{\prime}\right)$ for all $x \in R$ or $\mu(x) \geqslant \gamma(e)$ for all $x \in R^{\prime}$.

Proposition 4.9. Let $\mu=\mu_{1} \times \mu_{2} \times \cdots \times \mu_{n}$ and $\gamma=\gamma_{1} \times \gamma_{2} \times \cdots \times \gamma_{n}$ are fuzzy subsets of LA-rings $\mathrm{R}=\mathrm{R}_{1} \times \mathrm{R}_{2} \times \cdots \times \mathrm{R}_{\mathrm{n}}$ and $\mathrm{R}^{\prime}=\mathrm{R}_{1}^{\prime} \times \mathrm{R}_{2}^{\prime} \times \cdots \times \mathrm{R}_{\mathrm{n}}^{\prime}$ with left identities $\mathrm{e}=\left(\mathrm{e}_{1}, \mathrm{e}_{2}, \ldots, \mathrm{e}_{\mathrm{n}}\right)$ and $\mathrm{e}^{\prime}=$ $\left(e_{1}^{\prime}, e_{2} \prime, \ldots, e_{n}^{\prime}\right)$, respectively and $\mu \times \gamma$ is an anti fuzzy normal $L A$-sub-ring of an LA-ring $R \times R^{\prime}$. Then the following conditions are true.

1. If $\mu(x) \geqslant \gamma\left(e^{\prime}\right)$ for all $x \in R$, then $\mu$ is an anti fuzzy normal LA-sub-ring of $R$.

2. If $\mu(x) \geqslant \gamma(e)$ for all $x \in R^{\prime}$, then $\gamma$ is an anti fuzzy normal $L A$-sub-ring of $R^{\prime}$.

Proof.

1. Let $\mu(x) \geqslant \gamma\left(e^{\prime}\right)$, for all $x \in R$, and $y \in R$. We have to show that $\mu$ is an anti fuzzy normal LA-sub-ring of R. Now

$$
\begin{aligned}
\mu(x-y) & =\mu(x+(-y)) \\
& =\max \left\{\mu(x+(-y)), \gamma\left(e^{\prime}+\left(-e^{\prime}\right)\right)\right\} \\
& =(\mu \times \gamma)\left(x+(-y), e^{\prime}+\left(-e^{\prime}\right)\right) \\
& =(\mu \times \gamma)\left(\left(x, e^{\prime}\right)+\left(-y,-e^{\prime}\right)\right) \\
& =(\mu \times \gamma)\left(\left(x, e^{\prime}\right)-\left(y, e^{\prime}\right)\right) \\
& \leqslant(\mu \times \gamma)\left(x, e^{\prime}\right) \vee \mu \times \gamma\left(y, e^{\prime}\right)=\max \left\{\max \left\{\mu(x), \gamma\left(e^{\prime}\right)\right\}, \max \left\{\mu(y), \gamma\left(e^{\prime}\right)\right\}\right\} \\
& =\mu(x) \vee \mu(y),
\end{aligned}
$$


and

$$
\begin{aligned}
\mu(x y) & =\max \left\{\mu(x y), \gamma\left(e^{\prime} e^{\prime}\right)\right\} \\
& =(\mu \times \gamma)\left(x y, e^{\prime} e^{\prime}\right) \\
& =(\mu \times \gamma)\left(\left(x, e^{\prime}\right) \circ\left(y, e^{\prime}\right)\right) \\
& \leqslant(\mu \times \gamma)\left(x, e^{\prime}\right) \vee \mu \times \gamma\left(y, e^{\prime}\right) \\
& =\max \left\{\max \left\{\mu(x), \gamma\left(e^{\prime}\right)\right\}, \max \left\{\mu(y), \gamma\left(e^{\prime}\right)\right\}\right\} \\
& =\mu(x) \vee \mu(y) .
\end{aligned}
$$

Thus $\mu$ is an anti fuzzy LA-sub-ring of R. Now

$$
\begin{aligned}
\mu(x y) & =\max \left\{\mu(x y), \gamma\left(e^{\prime} e^{\prime}\right)\right\} \\
& =(\mu \times \gamma)\left(x y, e^{\prime} e^{\prime}\right) \\
& =(\mu \times \gamma)\left(\left(x, e^{\prime}\right) \circ\left(y, e^{\prime}\right)\right) \\
& =(\mu \times \gamma)\left(\left(y, e^{\prime}\right) \circ\left(x, e^{\prime}\right)\right) \\
& =(\mu \times \gamma)\left(y x, e^{\prime} e^{\prime}\right) \\
& =\max \left\{\mu(y x), \gamma\left(e^{\prime} e^{\prime}\right)\right\} \\
& =\mu(y x) .
\end{aligned}
$$

Hence $\mu$ is an anti fuzzy normal LA-sub-ring of R. 2 is same as 1 .

\section{References}

[1] M. T. Abu Osman, On the direct product of fuzzy subgroups, Fuzzy Sets and Systems, 12 (1984), 87-91. 1

[2] M. T. Abu Osman, On some product of fuzzy subgroups, Fuzzy Sets and Systems, 24 (1987), 79-86. 1

[3] S. Abou-Zaid, On normal fuzzy subgroups, J. Fac. Educ. Ain Shams Univ. Cairo., 13 (1988), 115-125. 1

[4] M. Akram, K. H. Dar, On anti fuzzy left h-ideals in hemirings, Int. Math. Forum, 2 (2007), 2295-2304. 1

[5] E. F. Alharfie, N. Muthana, The commutativity of prime rings with homoderivations, Int. J. Adv. Appl. Sci., 5 (2018), 79-81.

[6] R. Biswas, Fuzzy subgroups and anti fuzzy subgroups, Fuzzy Sets and Systems, 35 (1990), 121-124. 1

[7] J. R. Cho, J. Jezek, T. Kepka, Paramedial groupoids, Czechoslovak Math. J., 49 (1999), 277-290. 1

[8] K. A. Dib, N. Galhum, A. A. M. Hassan, Fuzzy rings and fuzzy ideals, Fuzzy Math., 4 (1996), 245-261. 1

[9] V. N. Dixit, R. Kumar, N. Ajmal, Fuzzy ideals and fuzzy prime ideals of a ring, Fuzzy Sets and Systems, 44 (1991), 127-138.

[10] K. C. Gupta, M. K. Kantroo, The intrinsic product of fuzzy subsets of a ring, Fuzzy Sets and Systems, 57 (1993), 103-110. 1

[11] S. M. Hong, Y. B. Jun, Anti fuzzy ideals in BCK-algebra, Kyungpook Math. J., 38 (1998), 145-150. 1

[12] J. Jezek, T. Kepka, Medial groupoids, Rozpravy CSAV Rada Mat. a Prir. Ved., 93 (1983), 93 pages. 1

[13] T. Kadir, In discrepancy between the traditional Fuzzy logic and inductive, Int. J. Adv. Appl. Sci., 1 (2014), 36-43.

[14] M. S. Kamran, Conditions for LA-semigroups to resemble associative structures, Ph.D. Thesis, Quaid-i-Azam University, Islamabad, (1993). 1

[15] N. Kausar, Direct product of finite intuitionistic anti fuzzy normal sub-rings over non-associative rings, Eur. J. Pure Appl. Math., 12 (2019), 622-648. 1

[16] N. Kausar, M. Alesemi, S. Salahuddin, M. Munir, A study on Ordered AG-groupoid by their fuzzy interior ideals, Int. J. Adv. Appl. Sci., 7 (2020), 75-82.

[17] N. Kausar, B. ul Islam, S. A. Ahmad, M. A. Waqar, Intuitionistics fuzzy ideals with thresholds $(\alpha, \beta]$ in LA-rings, Eur. J. Pure Appl. Math., 12 (2019), 906-943. 1

[18] N. Kausar, B. U. Islam, M. Y. Javaid, S. A. Ahmad, U. Ijaz, Characterizations of non-associative rings by the properties of their fuzzy ideals, J. Taibah. Univ. Sci., 13 (2019), 820-833. 1

[19] N. Kausar, M. A. Waqar, Characterizations of non-associative rings by their intuitionistic fuzzy bi-ideals, Eur. J. Pure Appl. Math., 12 (2019), 226-250. 1

[20] N. Kausar, M. A. Waqar, product of finite fuzzy normal sub-rings over non-associative rings, Int. J. Anal. Appl., 17 (2019), 752-770. 1

[21] M. A. Kazim, M. Naseeruddin, On almost semigroups, Aligarh Bull. Math., 2 (1972), 1-7. 1

[22] N. Kuroki, Regular fuzzy duo rings, Inf. Sci., 94 (1996), 119-139. 1 
[23] A. Lafi, DFIG control: A fuzzy approach, Int. J. Adv. Appl. Sci., 6 (2019), 107-116.

[24] W. J. Liu, Fuzzy invariant subgroups and ideals, Fuzzy Sets and Systems, 8 (1982), 133-139. 1

[25] T. K. Mukherjee, M. K. Sen, On fuzzy ideals of a ring I, Fuzzy Sets and Systems, 21 (1987), 99-104. 1

[26] T. K. Mukherjee, M. K. Sen, Prime fuzzy ideals in rings, Fuzzy Sets and Systems, 32 (1989), 337-341. 1

[27] M. Munir, N. Kausar, Salahuddin, Tehreem, On the prime fuzzy m-bi ideals in semigroups, J. Math. Computer Sci., 21 (2020), 357-365. 1

[28] P. V. Protić, N. Stevanović, AG-test and some general properties of Abel-Grassmann's groupoids, Pure Math. Appl., 6 (1995), 371-383. 1

[29] A. K. Ray, On product of fuzzy subgroups, Fuzzy sets and systems, 105 (1999), 181-183. 1

[30] T. Shah, N. Kausar, I. Rehman, Intuitionistic fuzzy normal sub-rings over a non-associative ring, An. Ştiinţ. Univ. "Ovidius" Constanţa Ser. Mat., 20 (2012), 369-386. 1, 3.1, 4.1

[31] T. Shah, I. Rehman, On LA-rings of finitely non-zero functions, Int. J. Contemp. Math. Sci., 5 (2010), 209-222. 1

[32] H. Sherwood, On product of fuzzy subgroups, Fuzzy Sets and Systems, 11 (1983), 79-89. 1

[33] U. M. Swamy, K. L. N. Swamy, Fuzzy prime ideals of rings, J. Math. Anal. Appl., 134 (1988), 94-103. 1

[34] L. A. Zadeh, Fuzzy sets, Information and control, 8 (1965), 338-353. 1 